\title{
Evaluation of the efficiency of artificial intelligence techniques of permeability results of a lower Albian carbonate reservoir of Campos Basin using the Winland method and cluster analysis
}

\author{
Mohammad Saad Al-lahham¹ \& Abel Carrasquilla', UENF/CCT/LENEP, Macaé - RJ, Brazil
}

\author{
${ }^{1}$ Petroleum Engineering and Exploration Laboratory, Darcy Ribeiro Northern Rio de Janeiro State University, Km 163 Amaral Peixoto \\ Highway, Brennand Avenue S/N, Imboassica, Macae-RJ, 27930-480.
}

Copyright 2019, SBGf - Sociedade Brasileira de Geofísica

This paper was prepared for presentation during the $16^{\text {th }}$ International Congress of the Brazilian Geophysical Society held in Rio de Janeiro, Brazil, 19-22 August 2019.

Contents of this paper were reviewed by the Technical Committee of the $16^{\text {th }}$ International Congress of the Brazilian Geophysical Society and do not necessarily represent any position of the SBGf, its officers or members. Electronic reproduction or storage of any part of this paper for commercial purposes without the written consent of the Brazilian Geophysical Society is prohibited.

\begin{abstract}
The selection of artificial intelligence (AI) techniques in the petroleum industry is very efficient because it will have a direct impact on the outcome of the study and the future use of the operation. In this study, we discuss the efficiency of the Al techniques by verification the results of permeability of a lower Albian carbonate reservoir of Campos Basin using the Winland method and cluster analysis. The permeability measured by $\mathrm{Al}$ techniques as fuzzy logic (FL), artificial neural network (ANN) and genetic algorithm (GA) were applied in three wells, being the first used for learning and the others as blind tests. Well logs are gamma ray, density, sonic, neutron porosity and SDR permeability logs. ANN obtained better performance compared to the $\mathrm{FL}$, but the results have become better with GA. Employing artificial intelligence (AI) modern techniques together with a dataset composed by well logs, lithological information and sample laboratory measurements of permeability and porosity.
\end{abstract}

\section{Introduction}

The term 'Artificial Intelligence' has become the most widely used in our time and has become one of the most active fields in scientific research all over the world. It has become clear that many of the possible applications through this field are not impossible but affordable, although require some patience and perseverance. This work aims at shedding light on Artificial Intelligence and its importance in the petroleum industry by reviewing its concept and its branches and verify the efficiency of estimated permeability results by Al techniques, which is considered one of the most important petrophysical properties of reservoirs and it is a measure of the ability of a porous material, often, a rock or an unconsolidated material, to allow fluids to pass through it. Winland method has been used as a useful evaluative tool by petrophysicists since the early 1970's and has also be used as a somewhat arbitrary "net pay cut-off". This is often done as a working guide to exclude very low porosity-permeability, using a slightly more scientific approach than simply selecting a certain porosity or permeability. Cluster analysis or clustering is the task of grouping a set of objects in such a way that objects in the same group (called a cluster) are more similar (in some sense) to each other than to those in other groups (clusters). Cluster analysis itself is not one specific algorithm, but the general task to be solved. It can be achieved by various algorithms that differ significantly in their understanding of what constitutes a cluster and how to efficiently find them. The permeability of a medium is linked to the porosity, but also to the shapes of the pores in the medium and their level of connectedness (Lucia, 2007). Justification of results by comparison of the results of each intelligent technique. Subsequently, an evaluation is made of how these techniques acted in the estimation of the permeability curves of SDR Permeability, using Mean Squared Normalized error (MSE), Coefficient of Determination $\left(\mathrm{R}^{2}\right)$, Winland (R35) methods and cluster analysis.

\section{Methodology}

The study was made via applying three methods of artificial intelligence: ANN (Graupe, 2013), FL (Chen \& Pham, 2001.) and GA (Coley,1999), through getting into four well logging data for wells 1,2 and 3. All these three wells belong to the Campos Basin. The well logs, which served as input to FL and ANN, were gamma ray, density, sonic and neutron porosity (Ellis \& Singer, 2007). In both cases, the permeability is the output parameter and GA serves as the optimizer of both estimates, since they enter as input to decrease the adjustment error. As targets for fit, permeability laboratory measurements in samples and in wells (SDR) were used. This last was derived from the nuclear magnetic resonance (NMR) log and proposed by Schlumberger Doll Research (Coates, et al., 1999.). As a criterion of the adjustment quality between the estimates and the targets, the Pearson correlation coefficient (R2) and Mean Square Error (MSE) were utilized. Justification the results using Winland R35 method and cluster analysis. In Winland's empirical relationship published by Kolodzie (1980) as:

$$
\log r_{35}=0.732+0.588 \log K_{\text {air }}-0.864 \log \phi_{\text {core }}
$$

where $r_{35}$ is the pore aperture radius corresponding to the 35th percentile of mercury saturation, $K_{\text {air }}$ is the uncorrected air permeability $(\mathrm{mD})$, and $\phi$ is porosity (\%). The highest statistical correlation was at the pore throat 
size corresponding to the 35th percentile of the cumulative mercury saturation curve, and this pore throat radius was named $\mathrm{R} 35$. The Wenland equation is applied in this work by used the laboratory data of the permeability and porosity, which are the real data of the reservoir. Data analysis using statistical methods using cluster method to present the accuracy of three well permeability results, cluster analysis method for dendrogram objects is provided for drawing hierarchical or tree-like structures, such as the results from clustering or a recursive partitioning regression tree. Therefore, gives the estimated results credibility and high accuracy. All the tests were performed using the modules present in Matlab (Matlab, 2016), R (statistical computing) and Interactive Petrophysics (IP, 2016) programs.

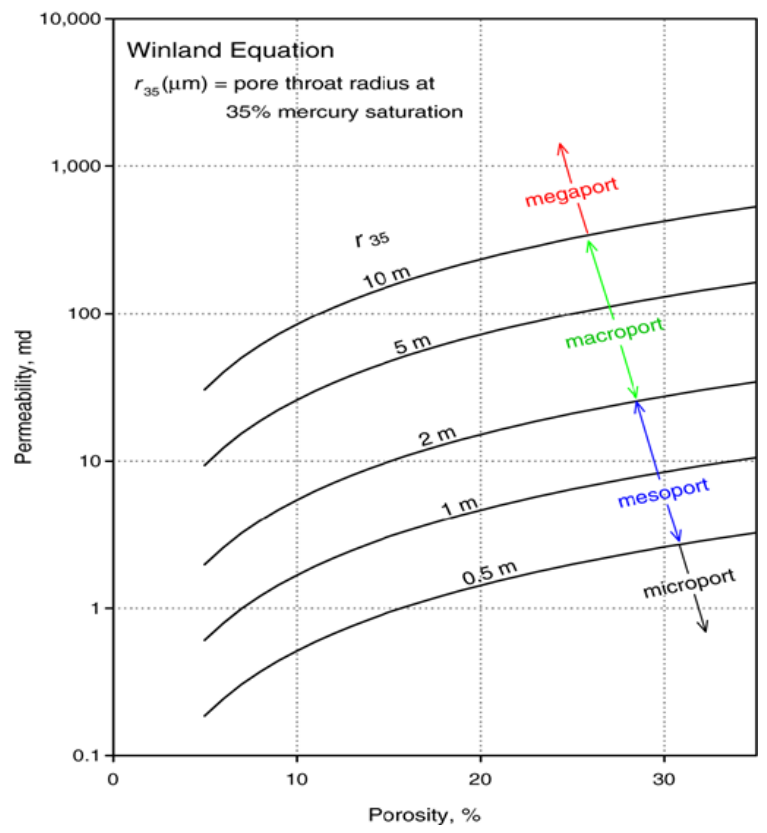

Figure 1 - Empirical model based on regression attributed to Winland, from (Kolodzie, 1980). Labels for four ranges of r35 are taken from (Martin et al, 1997).

\section{Results}

The Al technology was applied for the three wells, using ANN, FL and GA approaches and targeting the permeability of SDR, which is obtained in boreholes. In the ANN technique, Well 1 served as training and wells 2 and 3 as blind tests. Comparison of the estimates permeability obtained with the permeability of SDR using MSE and $R^{2}$ as indicative of quality for each technique. The Winland method presented pore diameter ranges as shown in figure 1, which can be classified into: macroporo, mesoporo and micropore, where the cluster analysis is used to present the accuracy of three well permeability results.

Thus, Figure 2, 3 and 4 shows in the left side, the adjustments obtained using the three techniques in well 1 , well 2 and well 3 with IP software, ANN (blue track), FL (green track) and GA (black track) approaches, by means of SDR permeability data (red track) as target, on the right side is the Winland curves of the laboratory data, in this case we have macropore in the blue area and mesopore in the green area, which are reflected to the results with the blue and green regions and can represented well within the results of the Al techniques.

Tables 1, 2 and 3 shows the fit errors, which provide a quantitative way to evaluate the performance of each approach. Generally, FL took first place, with GA in second and ANN in third, all using MSE criteria and in the $\mathrm{R}^{2}$ coefficient.

Using cluster analysis to enrich the accuracy of the results, we apply when we have too many data from many wells to analyze and study, the figure 5 shows the area of the results of $\mathrm{Al}$ techniques are used, which is the same lithology and zones in all of three wells, the Dendogram in figure 6 shows the results of the $\mathrm{Al}$ techniques using three and five blocks to see the accuracy and similarity of the results, which can be difficult see in MSE and $\mathrm{R}^{2}$ methods, in the first figure, the analysis is shown using three blocks of data inserted into three wells, where it was able to divide and analyze the data into three divisions, in the other figure, the analysis is shown using five blocks and was able to arrive at a more accurate analysis where the similarity results blocks were collected.

\section{Conclusions}

Through the application of artificial intelligence techniques to estimate permeability, satisfactory results were found in the present work, which can be considered as an important reference for the use of these techniques in the solution of related problems in the petroleum industry. The FL technique performed better compared to the ANN system, but the results became more satisfactory when using the GA method. However, each system achieved significant permeability approximations, proving the usefulness and reliability of these approaches. From the graphs of Winland presented, we can get the known pore diameter ranges, which can be classified into: macropore and mesopore through the data laboratory which is considered a real data and can be relied upon by comparison, blue region express micropore areas which has very low permeability which intersect with the low value in the estimated permeability results curves of the ANN, FL and GA, green region express mesopore areas which has higher value of permeability than micropore area that intersect with the higher value in the estimated permeability results curves of ANN, FL and GA, through this comparison using Winland we can say that the permeability results are quite satisfactory.

Using cluster analysis to enrich the accuracy of results, this analysis was able to divide the results into groups, with the application of more blocks we can come up with greater accuracy to compare the results, we can apply when have many results to analyze or have many wells to study. The results are thus encouraging because carbonates are very heterogeneous. 


\section{Acknowledgments}

We thank UENF / LENEP for the infrastructure, CNPq for the research grant, LR Senergy for the IP software license, and Petrobras / ANP for the dataset and research resources.

\section{References}

Bruhn, C.; Gomes, J.; Lucchese, C. \& Johann, P. 2003. Campos basin: reservoir characterization and management - historical overview and future challenges. Offshore Technology Conference. Paper OTC 15220.

Coates, G.; Xiao, L. \& Prammer, M. 1999. NMR logging. Principles \& Applications, Halliburton Energy Services Publication, Houston, 234 p.

Coley, D. 1999. An introduction to genetic algorithms for scientists and engineers. World Scientific Publishing Company, Singapore, $211 \mathrm{p}$.

Ellis, D. \& Singer, J. 2007. Well logging for earth scientists. Springer, Richmond, UK, 699 p.

Chen, G. \& Pham, T. 2001. Fuzzy sets, fuzzy logic, and fuzzy control systems. CRS Press, Boca Raton, Florida, 328 p.

Graupe, D. 2013. Principles of artificial neural networks. Advanced series in circuits and systems Vol. 7. World Scientific Publishing Co., Singapore, 363 p.

IP. 2016. Interactive Petrophysics Basic User's Guide, Norwegian University of Science and Technology, Oslo, $19 \mathrm{p}$.

Kolodzie Jr., S. 1980. Analysis of Pore Throat Size and Use of the Waxman-Smits Equation To Determine OOIP in Spindle Field, Colorado. Presented at the SPE Annual Technical Conference and Exhibition, Dallas, 21-24 September. SPE 9382.

Martin, A.J., Solomon, S.T., and Hartmann, D.J. 1997. Characterization of Petrophysical Flow Units in Five Carbonate Reservoirs. American Association of Petroleum Geologists Bull. 81 (5): 734.

Lucia, J. 2007. Carbonate reservoir characterization, an integrated approach. Springer-Verlag Berlin Heidelberg, $341 \mathrm{p}$.

Matlab. 2016. Matlab - the language of technical computing, Language reference manual, The MathWorks, Inc., Prime Park Way Natick, MA, $772 \mathrm{p}$.

VERMA, A. K.; CHEADLE, B. A.; ROUTRAY, A.; MOHANTY, W. K.; MANSINHA, L. Porosity and permeability estimation using neural network approach from well log data. In: SPE Annual Technical Conference and Exhibition. [S.I.: s.n.], 2012. p. 1-6.

Russell, S. \& Norvig, P. 20003. Artificial intelligence: a modern approach. Prentice Hall Series in Artificial Intelligence, Upper Saddle River, New Jersey, 1112 p. 

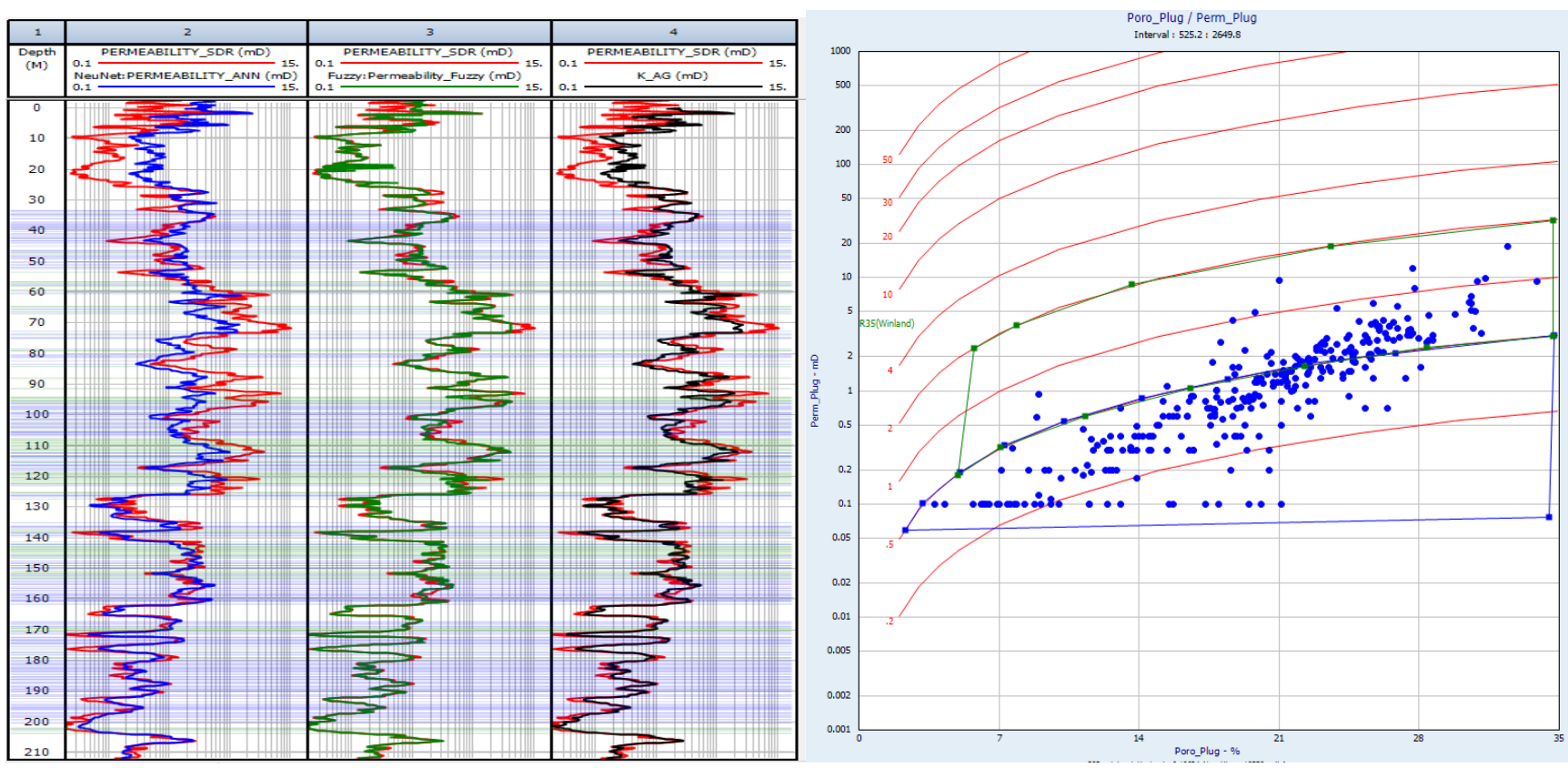

Figure 2 - Permeability estimates of well 1 using ANN (track 1, blue), FL (track 2, green) and GA (track 3, black) approaches, with SDR permeability (red) as target, the Winland curves of the laboratory data.

\begin{tabular}{|c|c|c|c|c|}
\hline \multirow{2}{*}{ Artificial Intelligence techniques } & \multicolumn{3}{|c|}{ Permeability of well 1 using IP software } \\
\cline { 2 - 5 } & MSE $(\mathrm{mD})^{2}$ & Classification & $\mathrm{R}^{2}$ & Classification \\
\hline Fuzzy Logic & 0.16169 & 1 & 0.9314 & 1 \\
\hline Artificial Neural Network & 1.2648 & 2 & 0.6376 & 3 \\
\hline Genetic Algorithm & 0.36646 & 3 & 0.9231 & 2 \\
\hline
\end{tabular}

Table 1 - Comparison between Al techniques results with SDR permeability as target of well 1.
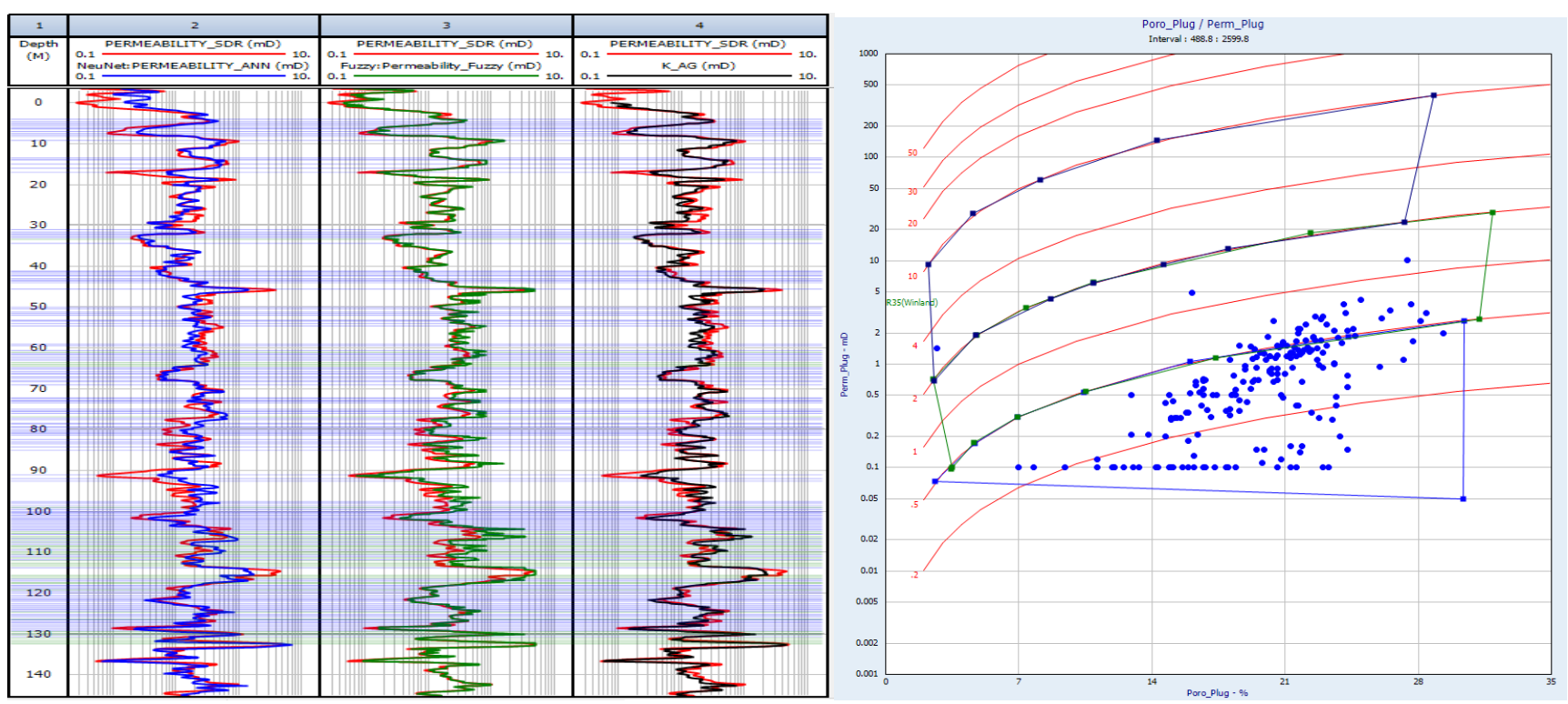

Figure 3 - Permeability estimates of well 2 using ANN (track 1, blue), FL (track 2, green) and GA (track 3, black) approaches, with SDR permeability (red) as target, the Winland curves of the laboratory data. 


\begin{tabular}{|c|c|c|c|c|}
\hline \multirow{2}{*}{ Artificial Intelligence techniques } & \multicolumn{3}{|c|}{ Permeability of well 2 using IP software } \\
\cline { 2 - 5 } & MSE $(\mathrm{mD})^{2}$ & Classification & $\mathrm{R}^{2}$ & Classification \\
\hline Fuzzy Logic & 0.11381 & 2 & 0.8909 & 1 \\
\hline Artificial Neural Network & 0.15001 & 3 & 0.8143 & 3 \\
\hline Genetic Algorithm & 0.070562 & 1 & 0.8874 & 2 \\
\hline
\end{tabular}

Table 2 - Comparison between AI techniques results with SDR permeability as target of well 2.
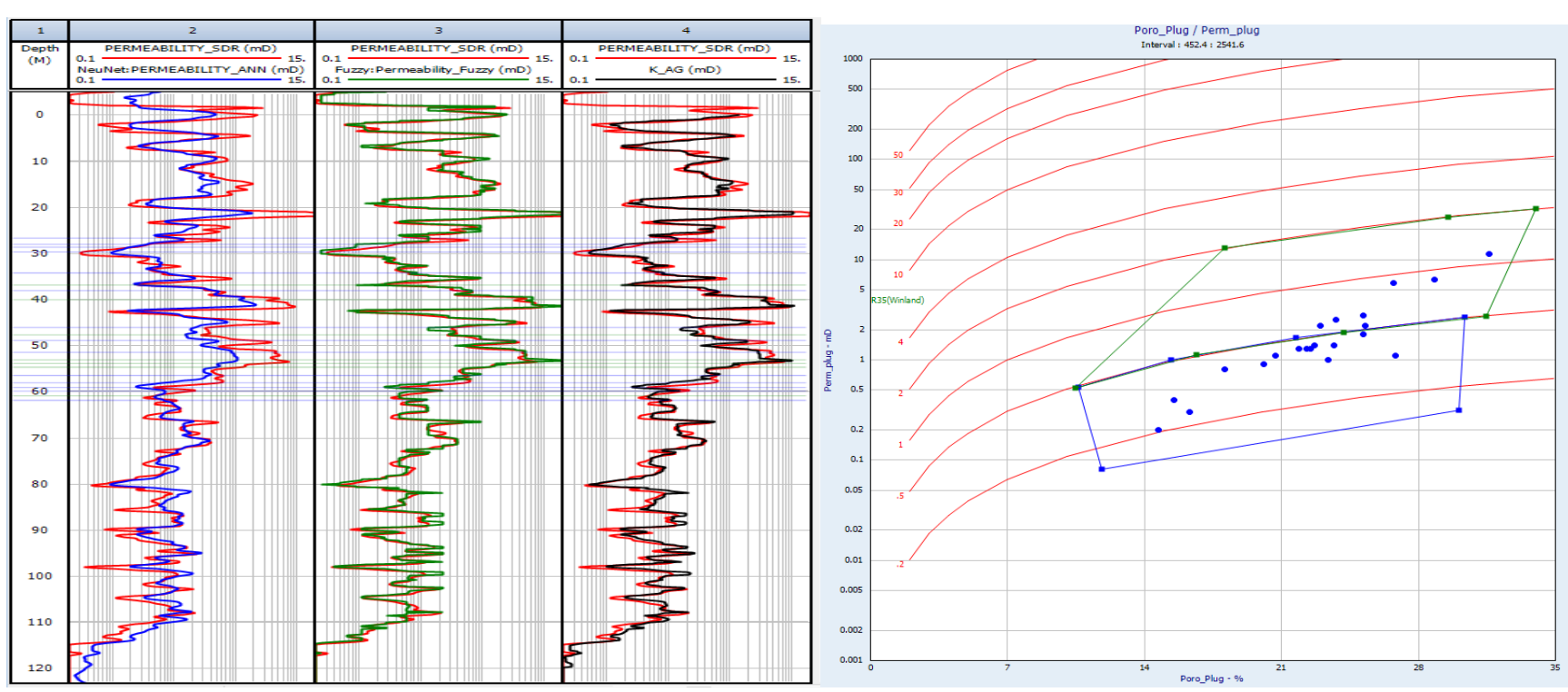

Figure 4 - Permeability estimates of well 3 using ANN (track 1, blue), FL (track 2, green) and GA (track 3, black) approaches, with SDR permeability (red) as target, the Winland curves of the laboratory data.

\begin{tabular}{|c|c|c|c|c|}
\hline \multirow{2}{*}{ Artificial Intelligence techniques } & \multicolumn{3}{|c|}{ Permeability of well X_3 using IP software } \\
\cline { 2 - 5 } & MSE $(\mathrm{mD})^{2}$ & Classification & $\mathrm{R}^{2}$ & Classification \\
\hline Fuzzy Logic & 1.4973 & 1 & 0.7668 & 1 \\
\hline Artificial Neural Network & 4.3908 & 2 & 0.5915 & 3 \\
\hline Genetic Algorithm & 3.8753 & 3 & 0.7320 & 2 \\
\hline
\end{tabular}

Table 3 - Comparison between Al techniques results with SDR permeability as target of well 3. 


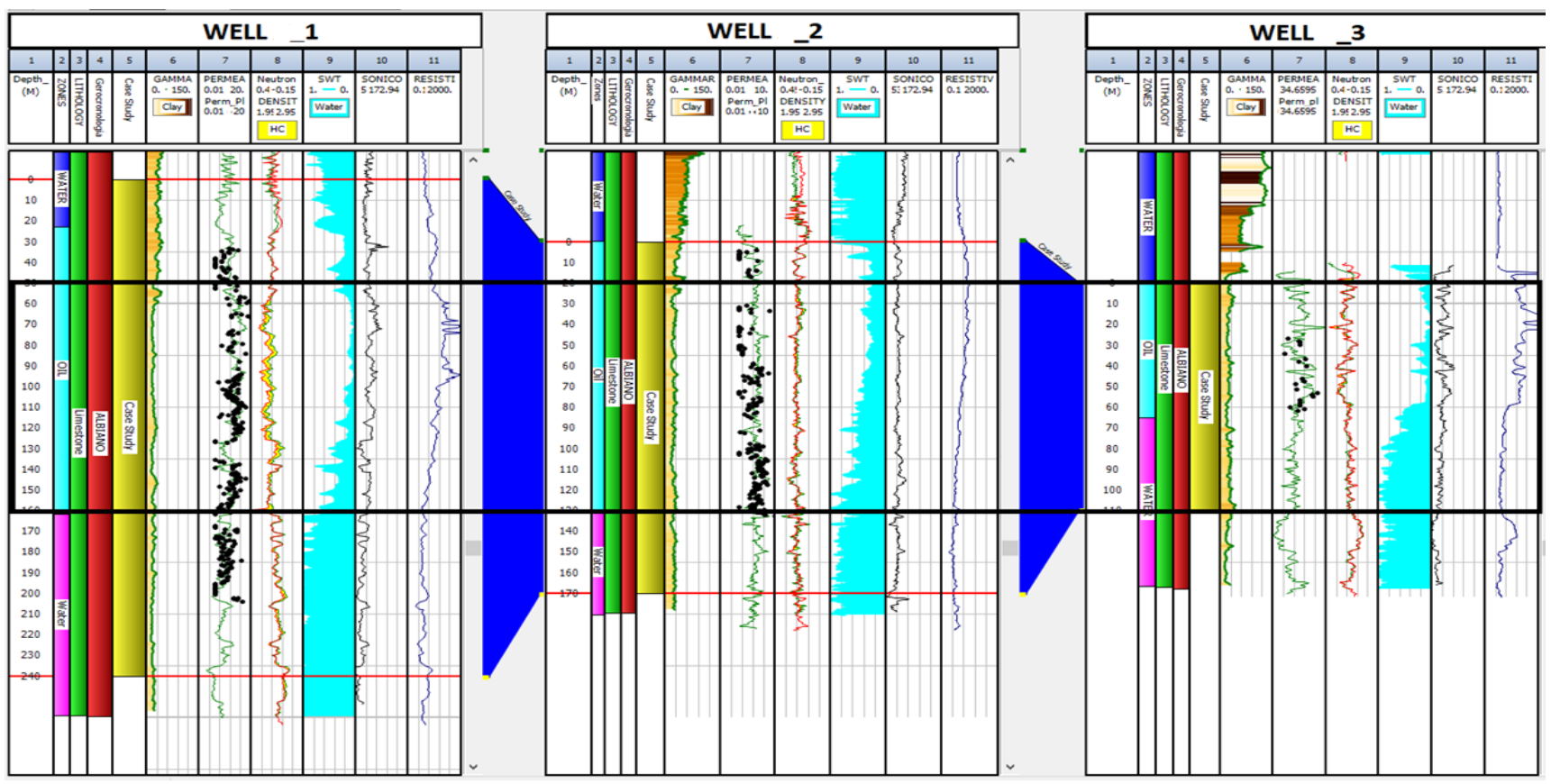

Figure 5 - Well logging data of wells 1,2 and 3

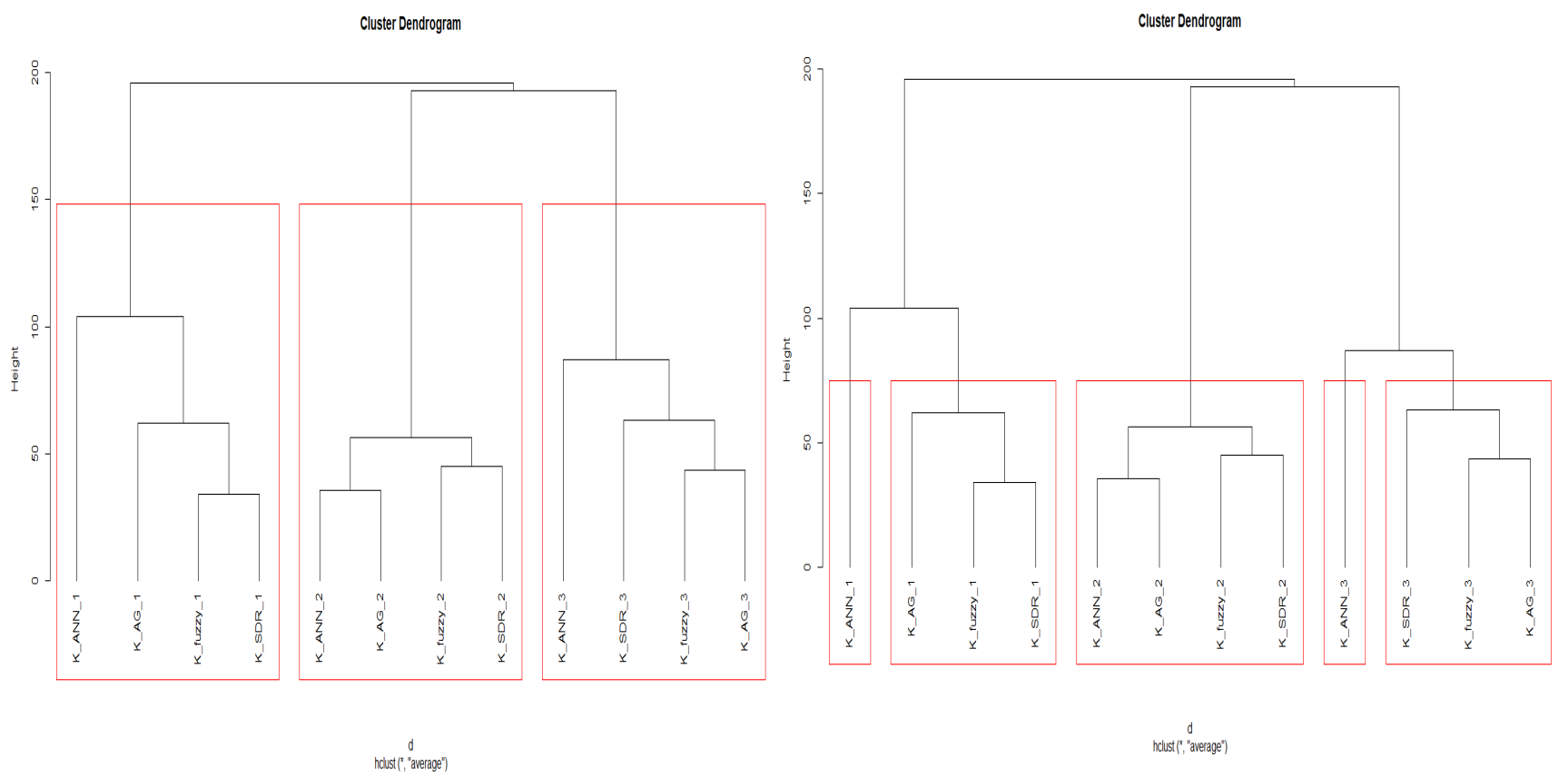

Figure 6 - Cluster Dendrogram analysis using the three/five blocks 